\title{
Bariatric surgery and incidental gastrointestinal stromal tumors - a single-center study
}

\author{
Maciej Walędziakk ${ }^{1}$, Anna Różańska-Walędziak ${ }^{2}$, Piotr K. Kowalewski ${ }^{1}$, Michał R. Janik ${ }^{1}$, Jakub Brągoszewski ${ }^{1}$, \\ Krzysztof Paśnik ${ }^{1}$ \\ ${ }^{1}$ Department of General, Oncological, Metabolic and Thoracic Surgery, Military Institute of Medicine, Warsaw, Poland \\ ${ }^{2} 2^{\text {nd }}$ Department of Obstetrics and Gynecology, Medical University of Warsaw, Warsaw, Poland
}

Videosurgery Miniinv 2017; 12 (3): 325-329

DOI: https://doi.org/10.5114/wiitm.2017.70215

\begin{abstract}
Introduction: Gastrointestinal stromal tumors (GISTS), originating from Cajal cells, are most commonly located in the stomach; therefore they can be found in the specimens excised during bariatric operations. The global prevalence of GISTs is about 130 cases per million population. Morbidity differs depending on geographical latitude. Although surgery is the treatment of choice for GISTS, 40-50\% of patients after radical surgical treatment will have a relapse or metastases.

Aim: To analyze the incidence of GISTs in patients undergoing bariatric surgery and to verify whether an operation performed according to the bariatric protocol is oncologically radical in case of GIST.

Material and methods: A single-center retrospective study. The study group comprised 1252 obese patients qualified for bariatric procedures, with no upper gastrointestinal tract neoplasms found during preoperative diagnostic examinations. In case of suspicious macroscopic pathologies $(n=81)$ present during the operation, tissue specimens underwent histopathological examination with further investigation performed if GISTS were found, including tumor size and localization, mitotic index and immunohistochemical analysis.

Results: Gastrointestinal stromal tumors were found in 16 cases, and benign tumors of various histological origin in 33 cases. All cases of GIST found came from stomach specimens, 7 from the gastric corpus vs. 9 from the fundus. Fourteen GISTs were found during laparoscopic sleeve gastrectomies (LSGS) vS. 2 during laparoscopic Roux-en-Y gastric bypasses (LRYGBS).

Conclusions: In case of incidental findings of GISTs during bariatric surgery, tumor resection with negative margins of incision may be considered as complete oncological treatment if there was very low/low risk stratification of GIST's recurrence after surgery.
\end{abstract}

Key words: obesity, bariatric surgery, gastrointestinal stromal tumor.

\section{Introduction}

Gastrointestinal stromal tumors (GISTs), mentioned for the first time in the 1980s, were classified as a separate group of tumors quite recently [1]. They probably originate from interstitial cells of Cajal [2], the pacemaker cells responsible for peristaltic contractions. Gastrointestinal stromal tumors most commonly occur in the gastrointestinal tract: the stomach (50-60\%) or small intestine (30-35\%), and less frequently the colon (5\%) or esophagus (1\%) [3]. Extra-gastrointestinal tract tumors (E-GISTs) constitute less than $5 \%$ of GISTs [4]. E-GISTs may also be metastases of tumors of unknown primary

\section{Address for correspondence}

Anna Różańska-Walędziak MD, PhD, $2^{\text {nd }}$ Department of Obstetrics and Gynecology, Medical University of Warsaw, 2 Karowa St,

00-315 Warsaw, Poland, phone: +48 606709 404, e-mail: aniaroza@tlen.pl 
location [3]. As Cajal cells are located mostly in the corpus and fundus of the stomach, these are also the most common locations of gastric GISTs [5]. The global prevalence of GISTs is about 130 cases per million population. Morbidity differs depending on geographical latitude, ranging from 7 new cases per million population a year in Europe and the United States to 16-22 cases in Asia [6]. Although surgery is the treatment of choice for GISTs, $40-50 \%$ of patients after radical surgical treatment will have a relapse or metastases [7].

Obesity has gradually become a common health problem, not only causing metabolic syndrome $[8,9]$, but also increasing the risk of developing neoplasms [10-12]. Bariatric surgery is the most effective way of treatment, exceeding in value diet or physical exercise or pharmacology [13-15].

More than 450000 bariatric procedures were performed globally in 2013, and the number is constantly growing every year [16]. The same trend may also be observed in Poland, where according to Janik [17], about 1500 bariatric operations were performed in 2014.

\section{Aim}

The purpose of the study was to analyze the incidence of GISTs in patients undergoing bariatric surgery.

We also wanted to verify whether an operation performed according to the bariatric protocol is oncologically radical in case of GIST.

\section{Material and methods}

In this retrospective study, medical records of 1252 patients qualified for bariatric procedures from January 2013 to December 2016 in a single large bariatric centre were analyzed. The qualification criteria were chosen as follows, according to current recommendations [18]: body mass index (BMI) greater than or equal to $40 \mathrm{~kg} / \mathrm{m}^{2}$, or BMI greater than or equal to $35 \mathrm{~kg} / \mathrm{m}^{2}$ with at least one serious obesity-related comorbidity, such as heart disease, type 2 diabetes mellitus (T2DM), or severe obstructive sleep apnea (OSA). Pre-operative examinations included basic blood tests, endoscopy of the upper gastrointestinal tract and ultrasound examination of the abdominal cavity. According to patients' BMI, alimentary habits and co-morbidities, they were qualified for sleeve gastrectomy (SG), duodenal switch (DS) or
Roux-en-Y gastric bypass (RYGB). The operative protocols were standardized and performed by the same team of bariatric surgeons. In case of any abnormal macroscopic findings during the operation samples were taken and a decision was made whether or not to proceed with the bariatric operation.

Further analysis was performed if GIST was found during the histopathological examination.

The analysis included age, gender, BMI, type of bariatric procedure, length of hospital stay (LOS), tumor size and localization, mitotic index (MI) and results of immunohistochemical investigation.

\section{Results}

In the study 1252 obese patients were qualified for bariatric surgery, none of whom had upper gastrointestinal tract neoplasms found during preoperative diagnostic examinations.

Out of $81(6.47 \%)$ patients from whom histopathological samples were collected, in $61 \%(n=49)$ cases the results were positive and showed abnormalities. After histological and immunohistochemical evaluation of stomach tissue samples, GISTs were diagnosed in $16(1.28 \%)$ patients, leiomyomas in $5(0.40 \%)$ patients, lipomas in $3(0.24 \%)$ patients, fibromas in $2(0.16 \%)$ patients, Schwannomas in $2(0.16 \%)$ patients and neurofibroma in $1(0.08 \%)$ patient. In 7 cases the procedure was discontinued after diagnostic laparoscopy and surgical biopsy, without performing the bariatric procedure. The incidental macroscopic findings, which led to waiving the bariatric procedure, originated from the liver (one of each: non-alcoholic steatosis cirrhosis and bile duct adenoma), stomach (one fibroma and one leiomyoma) and perigastric tissue (one of each: sarcoidosis and simple cyst).

Out of 16 patients, 10 (62.5\%) were male and $6(37.5 \%)$ were female. The mean age was $55.5 \pm 8.9$ years (range: 39 to 68 years). The mean BMI was $48.9 \pm 9.2 \mathrm{~kg} / \mathrm{m}^{2}\left(33.4-72.8 \mathrm{~kg} / \mathrm{m}^{2}\right)$. The mean length of stay in hospital was $4.7 \pm 1.0$ days ( 3 to 7 days). There were no severe complications or perioperative deaths.

All the cases of GIST found came from stomach specimens. Samples were taken during sleeve resection in 14 cases, and in 2 cases during Roux-en-Y gastric bypass.

All tumors were not larger than $20 \mathrm{~mm}$ in diameter. In 7 cases the tumors came from the gastric corpus, and 9 were found in the gastric fundus. 
In 12 cases the tumors were of single location, in 4 cases of double location. The results are shown in Table I.

In all the cases additional immunohistochemical examinations were performed, the results of which are shown in Table II, together with the mitotic count.

Most of the evaluated tumors were excised with negative margins of more than $10 \mathrm{~mm}$ (14 out of 16 cases), in 1 case the margin was of $5 \mathrm{~mm}$, and in 1 case the excision line was through the tumor. In the two latter cases the patients were invited to an oncological consultation, but they did not show up. No cases of local metastases to lymphatic nodes were found.

\section{Discussion}

All cases of GIST that were found in the study group were qualified as very low/low risk according

Table I. Characteristics of tumors

\begin{tabular}{|lcll|}
\hline Case & Size $[\mathrm{mm}]$ & $\begin{array}{c}\text { Type of } \\
\text { operation }\end{array}$ & Localization \\
\hline 1 & $10 \times 5$ & SLEEVE & Corpus \\
\hline 2 & $20 \times 15 \times 20$ & SLEEVE & Corpus \\
\hline 3 & 7 & SLEEVE & Fundus \\
\hline 4 & $5 \times 3$ & SLEEVE & Fundus \\
\hline 5 & 6 & RYGB & Fundus \\
\hline 6 & 5 & RYGB & Fundus \\
\hline 7 & $4 \times 3 \times 3$ & RYGB & Fundus \\
\hline 8 & 7 & SLEEVE & Corpus \\
\hline 9 & 6 & SLEEVE & Fundus \\
\hline 10 & $6 \times 4 \times 3$ & SLEEVE & Fundus \\
\hline 11 & 3 & SLEEVE & Corpus \\
\hline 12 & 4 & SLEEVE & Fundus \\
\hline 13 & 5 & SLEEVE & Fundus \\
\hline 14 & 5 & SLEEVE & Fundus \\
\hline 15 & 5 & SLEEVE & Fundus \\
\hline 16 & SLEEVE & Fundus \\
\hline & $5 L E E V E$ & Corpus \\
\hline 5 & SLEEVE & Corpus \\
\hline 12 & Corpus \\
\hline
\end{tabular}

to Modified National Institute of Health (NIH) consensus criteria [19] and in the $1^{\text {st }}$ group in the classification of the Armed Forces Institute of Pathology (AFIP) criteria [20], giving a 90-95\% chance of 10 years recurrence-free survival [3].

Morbid obesity not only leads to development of various metabolic co-morbidities, such as heart disease, diabetes mellitus type 2 or obstructive sleep apnea, but also may be a risk factor for developing neoplasms.

Bariatric surgery has become the most successful method of obesity treatment, especially in patients with co-morbidities resulting from the obesity itself. In many cases surgical treatment is the only way of physical and social recovery. The value of bariatric surgery has been confirmed in numerous studies.

Depending on patient's age and geographical latitude, global morbidity of GIST is 0.007-0.022 new cases per one thousand population a year, and in Europe about 0.01 new cases per one thousand population a year. These data come mostly from post mortem autopsies or incidental findings. In our study, we found 16 cases of GISTs in 1252 patients,

Table II. Immunohistochemical characteristics of tumors

\begin{tabular}{|c|c|c|c|c|c|c|c|}
\hline Case & CD117 & CD34 & SMA & Desmin & $\mathrm{S} 100$ & Ki67 & HPF \\
\hline 1 & + & & & & & & $0 / 50$ \\
\hline 2 & + & + & - & & & & $1 / 50$ \\
\hline 3 & + & + & - & & & & $0 / 50$ \\
\hline 4 & + & + & - & & & & $0 / 50$ \\
\hline 5 & + & + & $-1+$ & & - & & $1 / 50$ \\
\hline 6 & + & + & & - & & & $0 / 50$ \\
\hline 7 & + & + & - & & - & & $0 / 50$ \\
\hline 8 & + & + & + & & & + & $3 / 50$ \\
\hline 9 & - & + & - & & - & & $0 / 50$ \\
\hline 10 & + & + & - & & & & $0 / 50$ \\
\hline 11 & + & + & & & & & $0 / 50$ \\
\hline 12 & + & + & + & & & + & $1 / 50$ \\
\hline 13 & + & & - & & - & + & $5 / 50$ \\
\hline 14 & + & + & - & & - & & $1 / 50$ \\
\hline 15 & + & + & - & - & - & & $0 / 50$ \\
\hline 16 & + & + & - & & & & $0 / 50$ \\
\hline
\end{tabular}


during 47 months. Therefore the morbidity of GIST in our study is 12.8 new cases per 1 thousand population a year.

The results show that morbidity of GISTs found amongst patients undergoing bariatric surgery is 1280 times higher than in the general population. The results should be adjusted for the fact that most of the GISTs found during operations were small tumors (not larger than $20 \mathrm{~mm}$ ), which might be asymptomatic during patients' lifespan, and therefore might not be diagnosed.

There are several research studies about findings during bariatric surgery. Most GISTs found were at very low risk stratification following modified $\mathrm{NIH}$ criteria: Chiappetta et al. [21] found 0.31\% (8/2603) GISTs during RYGB and SG, Yuval et al. [22] reported 0.6\% (5/827) GISTs during SG, Sanchez et al. [23] found $0.8 \%$ (4/517) GISTs during RYGB, and Crouthamel et al. [24] found 0.8\% GISTs (12/1415) during SG, out of which only one was larger than $20 \mathrm{~mm}$.

In the Japanese study by Kawanowa et al. [25], 100 stomachs resected because of gastric cancer during the period 1988-2003 were analyzed and microGISTs were found in $35 \%$ of cases, of $0.2-4 \mathrm{~mm}$ in diameter. The histopathological examination was performed in the whole specimen, analyzing tissue slices sectioned at $5 \mathrm{~mm}$ intervals.

\section{Conclusions}

In case of incidental findings of GISTs during bariatric surgery, tumor resection with negative margins of incision may be considered as complete oncological treatment if there was very low or low risk stratification of GIST's recurrence after surgery.

All patients after GIST resection should remain under long-term postoperative care.

There is an important question whether obesity is a risk factor of GISTs, and further analysis must be conducted to confirm the answer.

\section{Conflict of interest}

The authors declare no conflict of interest.

\section{References}

1. Mazur MT, Brent HC. Gastric stromal tumors reappraisal of histogenesis. Am J Surg Pathol 1983; 7: 507-19.

2. Min KW. Gastrointestinal stromal tumor: an ultrastructural investigation on regional differences with considerations on their histogenesis. Ultrastruct Pathol 2010; 34: 174-88.
3. Heikki J, Vehtari A, Riihimäki J, et al. Risk of recurrence of gastrointestinal stromal tumour after surgery: an analysis of pooled population-based cohorts. Lancet Oncol 2012; 13: 265-74.

4. Stanek M, Pędziwiatr M, Matłok M, Budzyński A. Laparoscopic removal of gastrointestinal stromal tumors of uncinate process of pancreas. Videosurgery Miniinv 2015; 10: 311-5.

5. Yun HY, Sung R, Kim YC, et al. Regional distribution of interstitial cells of Cajal (ICC) in human stomach Korean. J Physiol Pharmacol 2010; 14: 317-24.

6. Joensuu H, Hohenberger P, Corless CL. Gastrointestinal stromal tumour. Lancet 2013; 382: 973-83.

7. Rutkowski P, Hompes D. Combined therapy of gastrointestinal stromal tumors. Surg Oncol Clin N Am 2016; 25: 735-59.

8. Hartemink N, Boshuizen HC, Nagelkerke NJ, et al. Combining risk estimates from observational studies with different exposure cutpoints: a meta-analysis on body mass index and diabetes type 2. Am J Epidemiol 2006; 163: 1042-52.

9. Landsberg L, Aronne LJ, Beilin LJ, et al. Obesity-related hypertension: pathogenesis, cardiovascular risk, and treatment: a position paper of The Obesity Society and the American Society of Hypertension. J Clin Hypertens (Greenwich) 2013; 15: 14-33.

10. Bhaskaran K, Douglas I, Forbes H, et al. Body-mass index and risk of 22 specific cancers: a population-based cohort study of 5.24 million UK adults. Lancet 2014; 384: 755-65.

11. Kaaks R, Lukanova A, Kurzer MS. Obesity, endogenous hormones, and endometrial cancer risk: a synthetic review. Cancer Epidemiol Biomarkers Prev 2002; 11: 1531-43.

12. Guh DP, Zhang W, Bansback N, et al. The incidence of co-morbidities related to obesity and overweight: a systematic review and meta-analysis. BMC Public Health 2009; 9: 88.

13. Colquitt JL, Pickett K, Loveman E, Frampton GK. Surgery for weight loss in adults. Cochrane Database Syst Rev 2014; 8 : CD003641.

14. Lindekilde N, Gladstone BP, Lübeck M, et al. The impact of bariatric surgery on quality of life: a systematic review and metaanalysis. Obes Rev 2015; 16: 639-51.

15. Gloy VL, Briel M, Bhatt DL, et al. Bariatric surgery versus non-surgical treatment for obesity: a systematic review and meta-analysis of randomised controlled trials. BMJ 2013; 347: f5934.

16. Angrisani L, Santonicola A, lovino P, et al. Bariatric surgery worldwide 2013. Obes Surg 2015; 25: 1822-32.

17. Janik MR, Stanowski E, Paśnik K. Present status of bariatric surgery in Poland. Videosurgery Miniinv 2016; 11: 22-5.

18. Neylan CJ, Kannan U, Dempsey DT, et al. The surgical management of obesity. Gastroenterol Clin North Am 2016; 45: 689-703.

19. Joensuu H. Risk stratification of patients diagnosed with gastrointestinal stromal tumor. Hum Pathol 2008; 39: 1411-9.

20. Miettinen M, Lasota J. Gastrointestinal stromal tumors: pathology and prognosis at different sites. Semin Diagn Pathol 2006; 23: $70-83$.

21. Chiappetta S, Theodoridou S, Stier C, Weiner RA. Incidental finding of GIST during obesity surgery. Obes Surg 2015; 25 : 579-83.

22. Yuval JB, Khalaileh A, Abu-Gazala M, et al. The true incidence of gastric GIST - a study based on morbidly obese patients undergoing sleeve gastrectomy. Obes Surg 2014; 24: 2134-7. 
23. Sanchez BR, Morton JM, Curet MJ, et al. Incidental finding of gastrointestinal stromal tumors (GISTs) during laparoscopic gastric bypass. Obes Surg 2005; 15: 1384-8.

24. Crouthamel MR, Kaufman JA, Billing JP, et al. Incidental gastric mesenchymal tumors identified during laparoscopic sleeve gastrectomy. Surg Obes Relat Dis 2015; 11: 1025-8.

25. Kawanowa K, Sakuma Y, Sakurai S, et al. High incidence of microscopic gastrointestinal stromal tumors in the stomach. Hum Pathol 2006; 37: 1527-35.

Received: 25.04.2017, accepted: 20.08.2017. 